\title{
On Effective Stochastic Generators for Conditioned Dynamics at an Atypical Reaction-Diffusion Current
}

\author{
Pegah Torkaman* and Farhad H. Jafarpou团 \\ Physics Department, Bu-Ali Sina University, 65174-4161 Hamedan, Iran
}

(Dated: June 28, 2018)

\begin{abstract}
We consider the fluctuations of a time-integrated particle current around an atypical value in a generic stochastic Markov process involving classical particles with two-site interaction and hardcore repulsion on a finite one-dimensional lattice with open boundaries. We address the question of which interactions one has to impose on such process to make the atypical value of the current typical. It is known that a corresponding effective stochastic Markov process might exist whose typical value of the current is equal to the atypical value of the current in the original process within a time-translational invariant regime. This effective process has, in principle, non-local transition rates. Nevertheless, it turns out that under some conditions the stochastic generator of the effective process has the same dynamical rules as the stochastic generator of the original process. We find these conditions and show that our approach can be generalized to any time-integrated observable.
\end{abstract}

PACS numbers: 05.40.-a,05.70.Ln,05.20.-y

Keywords: non-equilibrium systems, stochastic particle dynamics (theory), effective dynamics, current fluctuations, large deviations

\section{INTRODUCTION}

Rare events and their characterizations are of vital importance in different contexts of physics. These phenomena take place on a timescale much larger than the timescales characterizing the microscopic dynamics of the system. For example nucleation of crystals relies on a rare event i.e. the formation of the critical nucleus [1]. Protein-folding is also a rare event. In spite of an astronomical number of possible configurations for a protein, it folds into a unique native conformation [2]. Another classical example includes phase transformation for which the dynamics might be governed by rare events [3] .

In a general stochastic process the effective interactions that induce particular rare events are generally very complicated. In an equilibrium stochastic process the principle of detailed balance requires that the transition rates between a pair of microstates in the canonical ensemble of the process satisfy certain relation i.e. the ratio of rates for a transition and its time-reverse is given by the Boltzmann factor. As a sub-set of this equilibrium ensemble one can consider a particular driven ensemble consisting of phase-space paths (sometimes called an ensemble of trajectories) for which the mean flux of an observable on those paths is fixed. The existence of a net flux implies that the we are dealing with a constraint driven dynamics. Using the Bayes' theorem, it has been shown that the transition rates of this driven stochastic process with a given flux are related to those of the equilibrium system 4, 5]. Although unphysical transitions in the original equilibrium system, which might violate the relevant physical laws, will not appear in the driven dynamics;

\footnotetext{
*Electronic address: p.torkaman@basu.ac.ir
}

${ }^{\dagger}$ Electronic address: farhad@ipm.ir however, the transition rates of the driven system might be non-local. The non-equilibrium counterpart to the equilibrium detailed balance derived in [4, 5] results in a set of invariant quantities in the driven system analogues to the equilibrium one. This provides us with exact relations which help us calculate the transition rates in the driven system [6]. The results obtained in [4 6] can be reproduced by maximizing the dynamical entropy in the presence of appropriate constraints [7].

It is long known that in order to study the dynamics of a stochastic process conditioned on atypical values of a time-integrated observable in the steady state of a generic stochastic process system, whether this observable depends on microstates or transitions between a pair of microstates, one can use the concept of biased ensemble of trajectories [8]-[13]. This can be done by introducing a biasing field conjugated to the mean value of the observable. During a long observation time $t$ the ensemble average of a given observable in this biased ensemble of trajectories might depend on time and therefore, the time-translation invariance might be broken. However, there exists a time interval $\left[t_{1}, t_{2}\right]$, with $t_{1}$ and $t_{2}$ being far from the initial time and the final time 0 and $t$ respectively, where the time-translation invariance is held i.e. the ensemble average of the observable under investigation in this time interval is independent of time. It has been shown that being in the steady state and during this time-translational invariant regime $\left[t_{1}, t_{2}\right]$ the biased trajectories of the original process coincide with unbiased trajectories of an effective (or auxiliary) stochastic process [9]. Hence the average of the observable over the steady-state distribution of the effective stochastic process will be equal to its average over the biased ensemble of trajectories during the timetranslational invariant regime. The effective stochastic process is a conditioning-free process describing the problem of conditioning a Markov process on an atypical value 
of the dynamical observable. The mathematical relation between these processes is given by a generalization of Doob's h-transform [14]. It was shown that the effective process can be represented as a process satisfying various variational principles or a control process optimizing functionals related to the large deviations of the conditioning dynamical observable [15]. The connection between effective interactions and the theory of optimal control has also been studied in [16]. The analysis of the effective interaction in this way is used, for example, in the East model as one of the kinetically constrained models consisting of interacting spins in Glass-forming systems [17].

The effective stochastic process consists of those interactions one has to impose on the original stochastic process to make atypical behavior typical. As in the equilibrium case explained above, the effective process might be unphysical in the sense that its transition rates might be non-local [9]. This means that the original stochastic process and its corresponding effective stochastic process might not share similar features such as the range of interactions. The one-dimensional classical Ising chain, which exhibits ferromagnetic ordering in its biased ensemble of trajectories, is an example which reveals this feature [9]. Similar examples are studied in [17, 18].

A natural question that might arise is that under what conditions the corresponding effective stochastic process of a stochastic process with conditioned dynamics is physical in the sense that, in comparison with the original dynamics, no non-local transitions appear in the effective dynamics. In other words, under what conditions imposed on the microscopic reaction rates or for which atypical values of the observable, the stochastic generators of these two processes are exactly the same (up to a rescaling of the microscopic dynamical rules). This might not be valid for all atypical values of the observable; however, as we will see one might be able to find at least an atypical value of the observable for which the dynamics of the original stochastic process and its corresponding effective process share the same features at that point.

In present paper we are going to address the above question for a specific class of stochastic Markov processes consisting of interacting classical particles on a one-dimensional lattice with open boundaries. We assume that the particles are subjected to nearest-neighbor interactions in the bulk of the lattice while they can enter or leave the lattice from both the first and the last lattice sites. Considering the total reaction-diffusion current as a physical observable, we require that the corresponding effective stochastic process consists of exactly the same interactions in the bulk and at the boundaries of the lattice. In other words, we require that, up to a rescaling of the transition rates, the stochastic generator of the effective stochastic process is exactly the same as the stochastic generator of the original stochastic process conditioned on an atypical value of the total particle current. We show that, given that there are some constraints on the dynamical rules of the original stochastic process, there is at most a single atypical value of the average current at which this property might be held.

A couple of examples are given in the present paper. In the first example the Asymmetric Simple Exclusion Process (ASEP) is considered on an open lattice. In this system the particles with hard-core interactions perform a continuous-time simple random walk on an open lattice with the possibility of entering or leaving the lattice from both the first and the last lattice sites. Considering a barrier-free hopping of particles between the bulk of the lattice and the particle reservoirs with the same hopping rates as inside the bulk and assuming that the diffusion rates are biased to the right, it turns out that the conditions under which the effective and the original ASEP share the same features, restrict us to an atypical value of the particle current which is lower than the average particle current in the steady state of the original ASEP. On the other hand, it can be seen that the effective dynamics is exactly the one for the ASEP but with a reversed driving force (i.e. the diffusion rates are biased to the left). This phenomenon has already been observed in a recent work 10]. It has been shown that under some constraints the steady state of the effective ASEP can be written as a superposition of antishocks.

In the second example we consider an Asymmetric Kawasaki-Glauber Process (AKGP) on a onedimensional lattice with open boundaries [19]. In this case the non-zero rates are the death and branching rates as well as the hopping rate to the left. It is known that stable shocks can develop in the AKGP. Hence, a linear superposition of them can be used to construct its steady-state which consists of a hight-density phase and a low-density phase. As we will see by fine tuning the microscopic reaction rates the stochastic generator of the effective process can be brought to the form of the stochastic generator of the AKGP. Being in either of the static phases, the atypical current at which this phenomenon takes place can be lower or higher than the typical value of the current in the steady state, depending on the microscopic reaction rates.

Finally we will bring the third example in which the above mentioned phenomenon can happen for an atypical value of a non-entropic particle current. While the current in the ASEP is entropic and for the AKGP is zero (since the steady state is an equilibrium one), interestingly the large deviation function for the current in our third example satisfies the Gallavotti-Cohen-like symmetry [20]. On the other hand, the atypical value of the current at which the above mentioned phenomenon happens, is exactly equal to the typical value of the current in the steady state but with the opposite sign.

This paper is organized as follows. In section II we start with mathematical preliminaries and tools. In section III we define the reaction-diffusion current and find the conditions under which the stochastic generator of the effective process is equivalent with the stochastic generator of the original process. In section IV we will bring three examples to show how our constraints determine 
the effective dynamics. The generalization is brought in section $\mathrm{V}$. The last section is devoted to the outlook and conclusion.

\section{MATHEMATICAL TOOLS: A SHORT REVIEW}

We start with a stochastic Markov process in continuous-time. This is defined through a set of configurations denoted by $\{C\}$ and stochastic transition rates $\omega_{C \rightarrow C^{\prime}}$ between these configurations. Considering the complete basis vector $\{|C\rangle\}$, the probability of finding the system in configuration $C$ at time $t$ is given by $P(C, t)=\langle C \mid P(t)\rangle$ where the ket $|P(t)\rangle$ evolves in time according to the following master equation [21]

$$
\frac{d}{d t}|P(t)\rangle=\hat{\mathcal{H}}|P(t)\rangle
$$

in which the stochastic generator or Hamiltonian $\hat{\mathcal{H}}$ is a square matrix with the following matrix elements

$$
\left\langle C|\hat{\mathcal{H}}| C^{\prime}\right\rangle=\omega_{C^{\prime} \rightarrow C}-\delta_{C, C^{\prime}} \sum_{C^{\prime \prime} \neq C} \omega_{C \rightarrow C^{\prime \prime}} .
$$

Let us now consider a reaction-diffusion system consisting of interacting classical particles on a one-dimensional lattice of length $L$ which is modeled by a stochastic Markov process in continuous-time. Being in the steady state, we denote the mean (or typical) value of the reactiondiffusion current as $J^{*}$. Let $\mathcal{J}$ be the number of reaction and diffusion processes which contribute to the total reaction-diffusion current of the system up to the time $t$. This quantity is extensive with respect to $t$ and $L$. For a finite $L$ we define the space-time average of the total reaction-diffusion current as $J=\mathcal{J} /(L t)$ which is a time dependent quantity. During a long-time interval $t$ the probability to observe an atypical mean $J \neq J^{*}$ is exponentially small in $L$ and $t$. The large deviation property requires $P(\mathcal{J}) \propto \exp (-I(J) L t)$ where $I(J)$ is called the rate function. Now $\lim _{t \rightarrow \infty} \ln \left\langle e^{-s \mathcal{J}}\right\rangle /(L t)$ gives the cumulant generating function of the current $J$ in which $\left\langle e^{-s \mathcal{J}}\right\rangle=\sum_{\mathcal{J}} e^{-s \mathcal{J}} P(\mathcal{J})$ and that $s$ is called the counting field conjugated to the mean current $J[10,11]$.

We aim to study the dynamics of the above mentioned system conditioned on an atypical value of the current $J$. We define $\mathcal{J}_{C \rightarrow C^{\prime}}$ as an increment for this current during transition from configuration $C$ to $C^{\prime}$. It is known that the generating function of $\mathcal{J}$ defined above is given by $\left\langle e^{-s \mathcal{J}}\right\rangle=\left\langle\mathbb{1} \mid P_{s}(t)\right\rangle$ where $\langle\mathbb{1}|=\sum_{C}\langle C|$ is called the summation vector, and that $\left|P_{s}(t)\right\rangle$ should be obtained from the following master equation [22]

$$
\frac{d}{d t}\left|P_{s}(t)\right\rangle=\hat{\mathcal{H}}(s)\left|P_{s}(t)\right\rangle .
$$

The operator $\hat{\mathcal{H}}(s)$ in (2) is non-stochastic and called the modified Hamiltonian of the system with the following matrix elements

$$
\left\langle C|\hat{\mathcal{H}}(s)| C^{\prime}\right\rangle=e^{-s \mathcal{J}_{C^{\prime} \rightarrow C}} \omega_{C^{\prime} \rightarrow C}-\delta_{C, C^{\prime}} \sum_{C^{\prime \prime} \neq C} \omega_{C \rightarrow C^{\prime \prime}} .
$$

The counting field $s$ can be interpreted as a biasing field in the ensemble of dynamical trajectories which is sometimes called the $s$-ensemble. The role of $s$ in the dynamical ensemble is similar to the parameter $\beta$ (inverse of temperature) in the conventional equilibrium canonical ensemble. Using this biased ensemble one can study the dynamics of system during the observation time $t$ conditioned on a given value of the mean current $J$. Fixing some $s \neq 0$ correspond to studying those realizations of the process in which $J$ fluctuates around some atypical mean value [9]. This approach is sometimes called the grand canonical conditioning which corresponds to constructing a canonical ensemble of trajectories [10, 11, 13]. According to our notation the positive (negative) values of the counting field $s$ correspond to the atypical values of the current lower (higher) than the typical value of the current in the steady state. The time-evolution generator or modified Hamiltonian for the conditioned dynamics $\hat{\mathcal{H}}(s)$ is a non-stochastic operator which does not conserve probability. The sum of unnormalized probabilities is called the dynamical partition function of this dynamical ensemble and is given by $Z(s, t)=\left\langle\mathbb{1} \mid P_{s}(t)\right\rangle$. The logarithm of this quantity plays the role of the dynamical free energy of system which determines its dynamical phase behavior [8].

Following the discussion in section \, there is a timetranslational invariant regime during which one can construct an effective (or auxiliary) stochastic process whose unbiased dynamics produces the same value of mean current as the conditioned (or biased) dynamics explained above does during that time interval [9]. Considering the eigenvalue equations for the modified Hamiltonian $\hat{\mathcal{H}}(s)$

$$
\begin{aligned}
& \hat{\mathcal{H}}(s)|\Lambda(s)\rangle=\Lambda(s)|\Lambda(s)\rangle, \\
& \hat{\mathcal{H}}(s)\langle\tilde{\Lambda}(s)|=\Lambda(s)\langle\tilde{\Lambda}(s)|
\end{aligned}
$$

it has been shown that the stochastic generator of this effective stochastic process is given by [9]

$$
\hat{\mathcal{H}}_{e f f}(s)=\hat{U} \hat{\mathcal{H}}(s) \hat{U}^{-1}-\Lambda^{*}(s)
$$

in which $\hat{U}$ is a diagonal matrix with the matrix element $\langle C|\hat{U}| C\rangle=\left\langle\tilde{\Lambda}^{*}(s) \mid C\right\rangle$ and the asterisk stands for the largest eigenvalue and corresponding left and right eigenvectors of $\hat{\mathcal{H}}(s)$. The off-diagonal matrix elements of the operator $\hat{\mathcal{H}}_{\text {eff }}(s)$ in (3) are given by

$$
\left\langle C\left|\hat{\mathcal{H}}_{e f f}(s)\right| C^{\prime}\right\rangle=\frac{\left\langle\tilde{\Lambda}^{*}(s) \mid C\right\rangle\left\langle C|\hat{\mathcal{H}}(s)| C^{\prime}\right\rangle}{\left\langle\tilde{\Lambda}^{*}(s) \mid C^{\prime}\right\rangle} .
$$

It is easy to see that for the systems with a finite-dimensional configuration space $\Lambda^{*}(s)=$ $\lim _{t \rightarrow \infty} \ln \left\langle e^{-s \mathcal{J}}\right\rangle / t[22]$. 


\section{EQUIVALENCE OF ORIGINAL AND EFFECTIVE DYNAMICS}

In this section we limit ourselves to a family of singlespecies reaction-diffusion systems of classical particles with nearest-neighbor interactions in the bulk of a onedimensional lattice with open boundaries from there the particles can enter or leave the lattice. We aim to find the conditions under which the effective Hamiltonian $\hat{\mathcal{H}}_{e f f}(s)$ of this family is similar to that of the original process conditioned on some atypical mean current $J$ during its time-translational invariant regime, in the sense that the effective Hamiltonian consists of exactly the same type of interactions in the bulk and boundaries of the lattice. The simplest choice is where $\hat{U}$ in (3) is an identity matrix. This means that the modified Hamiltonian $\hat{\mathcal{H}}(s)$ and the effective Hamiltonian $\hat{\mathcal{H}}_{\text {eff }}(s)$ differ from each other by a constant which, according to (3), is the largest eigenvalue of the $\hat{\mathcal{H}}(s)$.

For the above mentioned family of stochastic processes the Hamiltonian $\hat{\mathcal{H}}$ can be written as

$$
\begin{aligned}
\hat{\mathcal{H}} & =\hat{\mathcal{L}} \otimes \mathcal{I}^{\otimes(L-1)} \\
& +\sum_{k=1}^{L-1}\left(\mathcal{I}^{\otimes(k-1)} \otimes \hat{h} \otimes \mathcal{I}^{\otimes(L-k-1)}\right) \\
& +\mathcal{I}^{\otimes(L-1)} \otimes \hat{\mathcal{R}}
\end{aligned}
$$

in which $\mathcal{I}$ is a $2 \times 2$ identity matrix. Introducing the basis kets

$$
|\emptyset\rangle=\left(\begin{array}{l}
1 \\
0
\end{array}\right),|A\rangle=\left(\begin{array}{l}
0 \\
1
\end{array}\right)
$$

in which $\emptyset$ and $A$ correspond to a vacancy and an occupied lattice site respectively, the matrix representation of $\hat{h}$ in the basis of $\{\emptyset \emptyset, \emptyset A, A \emptyset, A A\}$ and that of $\hat{\mathcal{L}}$ and $\hat{\mathcal{R}}$ in the basis of $\{\emptyset, A\}$ are given by

$$
\begin{gathered}
\hat{h}=\left(\begin{array}{llll}
\omega_{11} & \omega_{12} & \omega_{13} & \omega_{14} \\
\omega_{21} & \omega_{22} & \omega_{23} & \omega_{24} \\
\omega_{31} & \omega_{32} & \omega_{33} & \omega_{34} \\
\omega_{41} & \omega_{42} & \omega_{43} & \omega_{44}
\end{array}\right) \\
\hat{\mathcal{L}}=\left(\begin{array}{cc}
-\alpha & \gamma \\
\alpha & -\gamma
\end{array}\right), \hat{\mathcal{R}}=\left(\begin{array}{cc}
-\delta & \beta \\
\delta & -\beta
\end{array}\right) .
\end{gathered}
$$

The diagonal elements of $\hat{h}$ are given by $\omega_{i i}=$ $-\sum_{j \neq i} \omega_{j i}$. As can be seen the parameters $\alpha$ and $\gamma(\delta$ and $\beta$ ) are the injection and extraction rates of particles for the left (right) boundary respectively.

Let us consider the total reaction-diffusion current as the proper dynamical observable. The time-derivative of the average local density of particles is related to the average particle current through the following continuity equation

$$
\frac{d}{d t}\left\langle\rho_{k}\right\rangle=\left\langle j_{k-1}\right\rangle-\left\langle j_{k}\right\rangle+S_{k} \text { for } 1 \leq k \leq L
$$

in which $\left\langle j_{k}\right\rangle$ is defined as the average local particle current from the lattice site $k$ to $k+1$ and is given by

$$
\begin{aligned}
\left\langle j_{k}\right\rangle & =\left[\left(\omega_{21}-\omega_{31}\right)\left\langle\left(1-\rho_{k}\right)\left(1-\rho_{k+1}\right)\right\rangle\right. \\
& -\left(\omega_{12}+\omega_{42}+\omega_{32}\right)\left\langle\left(1-\rho_{k}\right) \rho_{k+1}\right\rangle \\
& +\left(\omega_{43}+\omega_{13}+\omega_{23}\right)\left\langle\rho_{k}\left(1-\rho_{k+1}\right)\right\rangle \\
& \left.+\left(\omega_{24}-\omega_{34}\right)\left\langle\rho_{k} \rho_{k+1}\right\rangle\right]\left(1-\delta_{k, L}\right)\left(1-\delta_{k, 0}\right) \\
& +\left(\beta\left\langle\rho_{k}\right\rangle-\delta\left\langle 1-\rho_{k}\right\rangle\right) \delta_{k, L} \\
& +\left(\alpha\left\langle 1-\rho_{k+1}\right\rangle-\gamma\left\langle\rho_{k+1}\right\rangle\right) \delta_{k, 0}
\end{aligned}
$$

for $k=0, \cdots, L . S_{k}$ is the source term. For the details of derivation (7) see Appendix. The average total reactiondiffusion current, which includes the contribution of all bonds of the lattice, is now given by

$$
\langle J\rangle=\frac{1}{L} \sum_{k=0}^{L}\left\langle j_{k}\right\rangle
$$

Considering the total reaction-diffusion current defined in (8) as a dynamical observable, the modified Hamiltonian $\hat{\mathcal{H}}(s)$ is given by

$$
\begin{aligned}
\hat{\mathcal{H}}(s) & =\hat{\mathcal{L}}(s) \otimes \mathcal{I}^{\otimes(L-1)} \\
& +\sum_{k=1}^{L-1}\left(\mathcal{I}^{\otimes(k-1)} \otimes \hat{h}(s) \otimes \mathcal{I}^{\otimes(L-k-1)}\right) \\
& +\mathcal{I}^{\otimes(L-1)} \otimes \hat{\mathcal{R}}(s)
\end{aligned}
$$

in which

$$
\begin{gathered}
\hat{h}(s)=\left(\begin{array}{cccc}
\omega_{11} & \omega_{12} e^{s} & \omega_{13} e^{-s} & \omega_{14} \\
\omega_{21} e^{-s} & \omega_{22} & \omega_{23} e^{-s} & \omega_{24} e^{-s} \\
\omega_{31} e^{s} & \omega_{32} e^{s} & \omega_{33} & \omega_{34} e^{s} \\
\omega_{41} & \omega_{42} e^{s} & \omega_{43} e^{-s} & \omega_{44}
\end{array}\right), \\
\hat{\mathcal{L}}(s)=\left(\begin{array}{cc}
-\alpha & \gamma e^{s} \\
\alpha e^{-s} & -\gamma
\end{array}\right), \hat{\mathcal{R}}(s)=\left(\begin{array}{cc}
-\delta & \beta e^{-s} \\
\delta e^{s} & -\beta
\end{array}\right) .
\end{gathered}
$$

The increment of the current for each reaction process or diffusion process can be understood from (7). Fixing the counting field $s$, corresponding to study of an atypical value of the current $\mathcal{J}$, and trying to find the effective Hamiltonian $\hat{\mathcal{H}}_{\text {eff }}(s)$ can be a formidable task.

Considering (4) one should note that $\hat{\mathcal{H}}_{\text {eff }}(s)$ can not necessarily be written in the two-site interaction form though $\hat{\mathcal{H}}$ is of the form (5). Generally speaking, a simple system might have complex effective interactions. As a matter of fact, it has been shown that even for a system with short-range interactions the effective interactions might be long-range [9, 17, 18]. However, as we will see, there might be a value of $s=s_{0}$ at which the stochastic Hamiltonian of effective dynamics is similar to (5) which means it involves nearest-neighbor interactions in the bulk and single-site interactions with the reservoirs at the boundaries. 
We have found that under the following constraints

$$
\begin{aligned}
e^{s_{0}} & =\frac{\omega_{13}+\omega_{23}+\omega_{43}-2 \omega_{21}}{\omega_{12}+\omega_{32}+\omega_{42}-2 \omega_{31}} \\
& =\frac{\omega_{13}+\omega_{23}+\omega_{43}-\omega_{21}-\alpha}{\gamma-\omega_{31}} \\
& =\frac{\beta-\omega_{21}}{\omega_{12}+\omega_{32}+\omega_{42}-\omega_{31}-\delta} \\
& =\frac{\omega_{24}-\omega_{21}}{\omega_{34}-\omega_{31}}>0
\end{aligned}
$$

the effective Hamiltonian $\hat{\mathcal{H}}_{\text {eff }}(s)$ has the form of (5) with

$$
\begin{aligned}
& \hat{h}_{e f f}\left(s_{0}\right)=\left(\begin{array}{cccc}
\omega_{11}^{\prime} & \omega_{12} e^{s_{0}} & \omega_{13} e^{-s_{0}} & \omega_{14} \\
\omega_{21} e^{-s_{0}} & \omega_{22}^{\prime} & \omega_{23} e^{-s_{0}} & \omega_{24} e^{-s_{0}} \\
\omega_{31} e^{s_{0}} & \omega_{32} e^{s_{0}} & \omega_{33}^{\prime} & \omega_{34} e^{s_{0}} \\
\omega_{41} & \omega_{42} e^{s_{0}} & \omega_{43} e^{-s_{0}} & \omega_{44}^{\prime}
\end{array}\right), \\
& \hat{\mathcal{L}}_{e f f}\left(s_{0}\right)=\left(\begin{array}{cc}
-\alpha e^{-s_{0}} & \gamma e^{s_{0}} \\
\alpha e^{-s_{0}} & -\gamma e^{s_{0}}
\end{array}\right), \\
& \hat{\mathcal{R}}_{e f f}\left(s_{0}\right)=\left(\begin{array}{cc}
-\delta e^{s_{0}} & \beta e^{-s_{0}} \\
\delta e^{s_{0}} & -\beta e^{-s_{0}}
\end{array}\right)
\end{aligned}
$$

where the diagonal elements of $\hat{h}_{e f f}$ are given by $\omega_{i i}^{\prime}=$ $-\sum_{j \neq i}\left(\hat{h}_{e f f}\left(s_{0}\right)\right)_{j i}$ which is the stochasticity condition for the effective Hamiltonian. The largest eigenvalue of $\hat{\mathcal{H}}(s)$ at $s=s_{0}$ turns out to be

$$
\begin{aligned}
\Lambda^{*}\left(s_{0}\right) & =\left(\alpha+(L-1) \omega_{21}\right)\left(e^{-s_{0}}-1\right) \\
& +\left(\delta+(L-1) \omega_{31}\right)\left(e^{s_{0}}-1\right)
\end{aligned}
$$

where its corresponding left eigenvector is given by $\left\langle\tilde{\Lambda}\left(s_{0}\right)\right|=\langle\mathbb{1}|$. One should note that the above left eigenvector results in the following exact expression for the generating function of the current at $s=s_{0}$

$$
\begin{aligned}
\left\langle e^{-s_{0} J}\right\rangle & =\left\langle\mathbb{1} \mid P_{s_{0}}(t)\right\rangle \\
& =\left\langle\mathbb{1}\left|e^{t \hat{\mathcal{H}}_{s_{0}}}\right| P_{s_{0}}(0)\right\rangle \\
& =e^{t \Lambda^{*}\left(s_{0}\right)}\left\langle\mathbb{1} \mid P_{s_{0}}(0)\right\rangle \\
& =e^{t \Lambda^{*}\left(s_{0}\right)} .
\end{aligned}
$$

Depending on the process under investigation, the eigenvalue (11) might depend linearly on the system size $L$. There are two cases for which the largest eigenvalue $\Lambda^{*}\left(s_{0}\right)$ can be independent of the system size. The first case is where $\omega_{21}=\omega_{31}=0$ while the rest of the reaction rules satisfy (10). In the second case $s_{0}=\ln \frac{\omega_{21}}{\omega_{31}}$ while

$$
\begin{aligned}
e^{s_{0}} & =\frac{\omega_{24}}{\omega_{34}}=\frac{\omega_{13}+\omega_{23}+\omega_{43}}{\omega_{12}+\omega_{32}+\omega_{42}} \\
& =\frac{\omega_{13}+\omega_{23}+\omega_{43}-\alpha}{\gamma} \\
& =\frac{\beta}{\omega_{12}+\omega_{32}+\omega_{42}-\delta}>0 .
\end{aligned}
$$

\section{EXAMPLES}

In this section a couple of examples are presented to show how the conditions obtained in the previous section might generate interesting results. In the first example we consider the ASEP with open boundaries as explained in the introduction. In the bulk of the lattice the particles hop to the right and left according to the following rules:

$$
\begin{aligned}
& A \emptyset \longrightarrow \emptyset A \quad \text { with the rate } \omega_{23}=p, \\
& \emptyset A \longrightarrow A \emptyset \quad \text { with the rate } \quad \omega_{32}=q
\end{aligned}
$$

All other reaction rates in the bulk of the lattice are zero. The particles are also injected and extracted from the boundaries of the lattice with the rates $\alpha, \gamma, \beta$ and $\delta$ as explained in the previous section. The constraints (10) give

$$
e^{s_{0}}=\frac{p}{q}, \frac{\alpha}{p}+\frac{\gamma}{q}=\frac{\beta}{p}+\frac{\delta}{q}=1 .
$$

Let us assume that the density of the particles at the left and right boundaries is kept fixed, using two particle reservoirs, at the values $\rho_{1}$ and $\rho_{2}$ respectively. This can be done by choosing barrier-free boundary rates defined as 10 ]

$$
\alpha=p \rho_{1}, \quad \gamma=q\left(1-\rho_{1}\right), \quad \beta=p\left(1-\rho_{2}\right), \quad \delta=q \rho_{2} .
$$

In this case the only constraint which remains will be

$$
e^{s_{0}}=\frac{p}{q}
$$

and the eigenvalue is given by

$$
\Lambda^{*}\left(s_{0}\right)=-(p-q)\left(\rho_{1}-\rho_{2}\right) .
$$

By substituting $s_{0}$ in $\hat{\mathcal{H}}(s)$ it is easy to see that the effective Hamiltonian can be obtained from the Hamiltonian of the original ASEP by exchanging $p$ and $q$ (or reversal $p \leftrightarrow q$ of the particle hopping rates). This observation has an interesting consequence. Given that one chooses barrier-free boundary rates, it is known that the steady state of the ASEP can be written as a linear superposition of Bernoulli measures with a step-function structure provided that the following constraint is satisfied [19]

$$
\frac{\rho_{2}\left(1-\rho_{1}\right)}{\rho_{1}\left(1-\rho_{2}\right)}=\frac{p}{q} .
$$

Hence, if one chooses $p>q$ then the constraint requires $\rho_{1}<\rho_{2}$ and this is what we call a shock structure. Now, since the effective Hamiltonian is exactly the same as the original Hamiltonian but with reversed hopping rates one can conclude that the steady state of the effective Hamiltonian can also be written as a superposition of Bernoulli measures with a step-function structure. In this case one should have

$$
\frac{\rho_{2}\left(1-\rho_{1}\right)}{\rho_{1}\left(1-\rho_{2}\right)}=\frac{q}{p}
$$


and since $p>q$ then $\rho_{1}>\rho_{2}$. In comparison to the definition of a shock structure this is called an antishock. One should note that since for $p>q$ we have $s_{0}>0$, then atypical value of the current is always lower than the typical value in the steady state. This has already been observed and discussed with more detail in [10].

In the second example we consider an asymmetric Kawasaki-Glauber process which contains the following reaction rules in the bulk of the lattice:

$$
\begin{aligned}
& \emptyset A \longrightarrow \emptyset \emptyset \text { with the rate } \omega_{12}, \\
& \emptyset A \longrightarrow A \emptyset \text { with the rate } \omega_{32}, \\
& \emptyset A \longrightarrow A A \text { with the rate } \omega_{42} \\
& A \emptyset \longrightarrow \emptyset \emptyset \text { with the rate } \omega_{13} \\
& A \emptyset \longrightarrow A A \text { with the rate } \omega_{43} .
\end{aligned}
$$

The only non-zero boundary rates $\alpha$ and $\beta$ define the injection and extraction of the particles at the left and right boundaries of the lattice respectively. The constraints (10) for this process are

$$
e^{s_{0}}=\frac{\omega_{13}+\omega_{43}}{\omega_{12}+\omega_{42}+\omega_{32}}, \quad \alpha=\beta=\omega_{13}+\omega_{43} .
$$

It is known that the steady state of this process, without any constraints on the microscopic reaction rates, can be written as a superposition of stable Bernoulli shock measures [19]. It has also been shown that the microscopic position of each shock performs a biased random walk on the lattice. Now, following our discussion in the first example, we conclude that the steady state of the effective dynamics can be written in terms of superposition of Bernoulli shock measures (and not antishocks). Note that in the steady state of the original process the system undergoes a static phase transition between a low-density and a high-density phase depending on the values of $\omega_{13}$ and $\omega_{43}$. It is worth mentioning that, being in either of these static phases, the dynamics can be either conditioned on a lower than typical or a higher than typical value of the total average current.

The time-integrated currents are generally either entropic which satisfy the Gallavotti-Cohen symmetry such as the one studied in 23] or non-entropic which satisfy the Gallavotti-Cohen-like symmetry such as the one studied in [20]. In the third example we introduce a non-entropic reaction-diffusion current which satisfies the Gallavotti-Cohen-like symmetry with the mentioned property. It turns out that under some constraints the value of the conjugated field $s_{0}$ can be located on the symmetry point corresponding to $s=0$ which means we have $\Lambda^{*}(0)=\Lambda^{*}\left(s_{0} \neq 0\right)=0$. This indicates that the absolute values of the atypical current and the typical current are equal; however, they flow in opposite directions.

Our third example consists of birth and death processes in the bulk of the lattice with the rates $\left(\omega_{21}, \omega_{31}\right)$ and $\left(\omega_{12}, \omega_{13}\right)$ respectively which can be demonstrated as

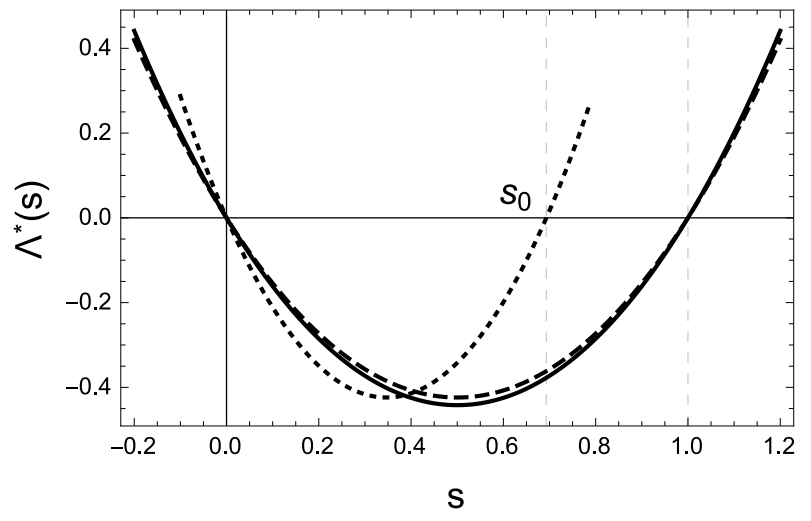

FIG. 1: The plot of the numerically calculated largest eigenvalue of the modified Hamiltonian. The dotted curve is $\Lambda_{\text {cur }}^{*}(s)$ for $\omega_{12}=1, \omega_{13}=2, \omega_{21}=0.8$ for a lattice of length $L=6$. The solid line and the dashed line correspond to $\Lambda_{\text {ent }}^{*}(s)$ and $\Lambda_{\text {cur }}^{*}(E s)$ respectively. The vertical line is $s_{0}=\ln \left(\omega_{13} / \omega_{12}\right)=0.69$. See inside the text for more information.

follows:

$$
\begin{aligned}
& \emptyset \emptyset \longrightarrow \emptyset A \text { with the rate } \omega_{21}, \\
& \emptyset \emptyset \longrightarrow A \emptyset \text { with the rate } \omega_{31}, \\
& \emptyset A \longrightarrow \emptyset \emptyset \text { with the rate } \omega_{12}, \\
& A \emptyset \longrightarrow \emptyset \emptyset \text { with the rate } \omega_{13}
\end{aligned}
$$

The particles are allowed to enter or leave the lattice from both boundaries; however, the boundary rates $\alpha$ and $\delta$ are assumed to satisfy the following constraints

$$
\alpha=\omega_{21}, \delta=\omega_{31} .
$$

Now the constraints (10) lead us to

$$
\begin{aligned}
& s_{0}=\ln \frac{\omega_{21}}{\omega_{31}}=\ln \frac{\omega_{13}}{\omega_{12}}, \\
& \gamma=\omega_{12}-\omega_{31}, \\
& \beta=\omega_{13}-\omega_{21} .
\end{aligned}
$$

The largest eigenvalue of the modified Hamiltonian for the entropy production $\Lambda_{\text {ent }}^{*}(s)$ and that of the total diffusion-reaction current $\Lambda_{c u r}^{*}(s)$ are numerically calculated and plotted in FIG[1. We have also plotted $\Lambda_{\text {cur }}^{*}(E s)$ where $E=s_{0}$. As can be seen $\Lambda_{\text {ent }}^{*}(s)$ does not lie on $\Lambda_{c u r}^{*}(E s)$ and therefore the total diffusion-reaction current is non-entropic [20].

\section{GENERALIZATIONS}

The above discussion can be generalized to any arbitrary time-integrated observable (which is not necessarily the particle current) in a continuous-time stochastic Markov process with a stochastic generator of type (5) and a finite configuration space. These observables can be fluxes or currents which depend on the transitions between configurations or microstates, such as the one 
we explained in this paper. Alternatively we can consider those time-integrated observables that might have merely a spatial nature such as dynamical activity [8] or energy 9].

For the fluxes or currents which are defined on the basis of transitions between configurations, we consider the increment $\theta_{C \rightarrow C^{\prime}}$ whenever the system jumps from $C$ to $C^{\prime}$ along a spatio-temporal trajectory. For the dynamical activity one has $\theta_{C \rightarrow C^{\prime}}=1$ for all $C$ and $C^{\prime}\left(C \neq C^{\prime}\right)$ while for the entropy production the increment will be $\theta_{C \rightarrow C^{\prime}}=\ln \left(\omega_{C \rightarrow C^{\prime}} / \omega_{C^{\prime} \rightarrow C}\right)$ [24]. We have already defined the increments for a global reaction-diffusion current in section III. These increments, as we saw, affect the non-diagonal elements of the modified Hamiltonian. In contrast, for those time-dependent observables which are defined along a spatio-temporal trajectory and depend on the visited microstates, only the diagonal elements of the modified Hamiltonian, depending on the observable, are changed.

In either of these two cases we start with constructing the modified Hamiltonian $\hat{\mathcal{H}}(s)$ for the observable under investigation. Let us denote the sum of the matrix elements of $i$ th column of $\hat{h}(s)$ as $h_{i}$ for $i=1, \cdots, 4$. For $\hat{\mathcal{R}}(s)$ and $\hat{\mathcal{L}}(s)$ they will be denoted by $r_{i}$ and $l_{i}$ respectively for $i=1,2$. It can be shown that the summation vector $\langle\mathbb{1}|$ is the left eigenvector of the modified Hamiltonian given that

$$
h_{3}-h_{1}=h_{1}-h_{2}=l_{1}-l_{2}=r_{2}-r_{1}, \quad h_{4}=h_{1} .
$$

At the same time the eigenvalue of the modified Hamiltonian associated with that left eigenvector is given by

$$
\Lambda^{*}(s)=l_{1}+r_{1}+(L-1) h_{1}
$$

in which $L$ is the size of the lattice. The equations (20) determine the value(s) of the conjugated field $s_{0}$ and also the probable constraints on the microscopic reaction rates under which the original and the effective dynamics are equivalent in the sense of what was explained in section III

\section{CONCLUDING REMARKS}

In order to investigate the dynamics of a generic stochastic Markov process conditioned on an atypical value of an integrated current during its timetranslational invariance regime, one can modify its stochastic generator to build an effective (or auxiliary) stochastic generator for which the typical value of the integrated current in the steady state is equal to the atypical value of the integrated current in the original process. However, one realizes that the resulting effective process might be unphysical in the sense that it might contain non-local transitions. In this paper we have shown that under some constraints on the microscopic reaction rates, the stochastic generator of the effective stochastic process can posses exactly the same dynamical rules as the original process does, at least for a specific value of the current under investigation. We have also shown that, depending on the process, this current might be entropic or non-entropic. Possible generalizations have also been discussed. Our approach might not be the only possible way to construct such effective stochastic process who shares identical features with the original stochastic process. It would be of great interest if one could find the general conditions under which the effective process would be physical in the sense that it only contains local transitions. On the other hand, we only considered the reaction-diffusion processes with nearest-neighbor interactions on open lattices. It would be interesting to investigate the processes with long-range interactions, not only on an open lattice but under periodic boundary conditions.

\section{Appendix: Derivation of the particle current formula (7)}

The time evolution of the average local particle density $\left\langle\rho_{k}\right\rangle(t)$ at the lattice site $k$ at time $t$ is given by

$$
\frac{d}{d t}\left\langle\rho_{k}\right\rangle=J_{k-1 \rightarrow k}^{R}+J_{k \leftarrow k+1}^{R}+J_{k-1, k}^{D}-J_{k, k+1}^{D}
$$

for $k=1,2, \ldots, L$ where $J_{k-1 \rightarrow k}^{R}$ and $J_{k \leftarrow k+1}^{R}$ are the average input current into the lattice site $k$, in the result of reaction with the lattice sites $k-1$ and $k+1$ respectively. $J_{k, k+1}^{D}$ and $J_{k-1, k}^{D}$ are also the net average diffusion current from the lattice site $k$ to $k+1$ and from $k-1$ to $k$ respectively. Note that $J_{0 \leftrightarrows 1}^{R}=J_{L \leftrightarrows L+1}^{R}=0$ while $J_{0,1}^{D}=J_{L, L+1}^{D} \neq 0$ which give the particle exchange with particle reservoirs at the boundaries. These quantities are given by

$$
\begin{aligned}
J_{k-1 \rightarrow k}^{R} & =\left[\left(\omega_{21}+\omega_{41}\right)\left\langle\left(1-\rho_{k-1}\right)\left(1-\rho_{k}\right)\right\rangle\right. \\
& -\omega_{12}\left\langle\left(1-\rho_{k-1}\right) \rho_{k}\right\rangle+\omega_{43}\left\langle\rho_{k-1}\left(1-\rho_{k}\right)\right\rangle \\
& \left.-\left(\omega_{14}+\omega_{34}\right)\left\langle\rho_{k-1} \rho_{k}\right\rangle\right]\left(1-\delta_{k, 1}\right) \\
J_{k \leftarrow k+1}^{R} & =\left[\left(\omega_{31}+\omega_{41}\right)\left\langle\left(1-\rho_{k}\right)\left(1-\rho_{k+1}\right)\right\rangle\right. \\
& +\omega_{42}\left\langle\left(1-\rho_{k}\right) \rho_{k+1}\right\rangle-\omega_{13}\left\langle\rho_{k}\left(1-\rho_{k+1}\right)\right\rangle \\
& \left.-\left(\omega_{14}+\omega_{24}\right)\left\langle\rho_{k} \rho_{k+1}\right\rangle\right]\left(1-\delta_{k, L}\right)
\end{aligned}
$$

for $k=1,2, \ldots, L$ and

$$
\begin{aligned}
J_{k, k+1}^{D} & =\omega_{23}\left\langle\rho_{k}\left(1-\rho_{k+1}\right)\right\rangle\left(1-\delta_{k, L}\right)\left(1-\delta_{k, 0}\right) \\
& -\omega_{32}\left\langle\left(1-\rho_{k}\right) \rho_{k+1}\right\rangle\left(1-\delta_{k, L}\right)\left(1-\delta_{k, 0}\right) \\
& +\left(\beta\left\langle\rho_{k}\right\rangle-\delta\left\langle 1-\rho_{k}\right\rangle\right) \delta_{k, L} \\
& +\left(\alpha\left\langle 1-\rho_{k+1}\right\rangle-\gamma\left\langle\rho_{k+1}\right\rangle\right) \delta_{k, 0}
\end{aligned}
$$

for $k=0, \cdots, L$. The average local density of particles is related to the average particle current through the following continuity equation (6). Comparing (A.1) and (6) 
one finds the following relations for the average particle current $\left\langle j_{k}\right\rangle$ and the source term $S_{k}$

$$
\begin{aligned}
& \left\langle j_{k}\right\rangle=J_{k \rightarrow k+1}^{R}-J_{k \leftarrow k+1}^{R}+J_{k, k+1}^{D}, \\
& S_{k}=J_{k-1 \leftarrow k}^{R}+J_{k \rightarrow k+1}^{R} .
\end{aligned}
$$

[1] S. Auer and D. Frenkel, Nature 409, 6823 (2001); R. P. Sear, J. Phys.: Cond. Matt. 19 033101(2007)

[2] E. I. Shakhnovich, A. M. Gutin, Nature 346773 (1990); E. I. Shakhnovich, Chem. Rev. 1061559 (2006); G. D. Rose, P. J. Fleming, J. R. Banavar, and A. Maritan, Proc. Natl. Acad. Sci. USA 10316623 (2006).

[3] E. Weinan, R. Weiqing and E. Vanden-Eijnden, J. Phys. Chem. B 1096688 (2005)

[4] R. M. L. Evans, Phys. Rev. Lett. 92150601 (2004)

[5] R. M. L. Evans, J. Phys. A: Math. Gen. 38293 (2005)

[6] A. Baule, R. M. L. Evans, Phys. Rev. Lett 101240601 (2008)

[7] C . Monthus, J. Stat. Mech. P03008 (2011)

[8] V. Lecomte, C. Appert-Rolland and F. van Wijland, J. Stat. Phys. 12751 (2007); J. P. Garrahan, R. L. Jack, V. Lecomte, E. Pitard, K. van Duijvendijk and F. van Wijland, J. Phys. A: Math. Theor. 42075007 (2009)

[9] R. L. Jack, P. Sollich, Prog. Theor. Phys. Supp. 184304 (2010)

[10] V. Belitsky, G. M. Schütz, J. Phys. A: Math. Theor. 46 295004 (2013)

[11] V. Belitsky, G. M. Schütz, J. Stat. Phys. 15293 (2013)

[12] R. J. Harris, V. Popkov, G. M. Schütz, Entropy 155065
(2013)

[13] V. Popkov, G. M. Schütz, and D. Simon, J. Stat. Mech. P10007 (2010)

[14] R. Chetrite, H. Touchette, arXiv:1506.05291 (2015)

[15] R. Chetrite, H. Touchette, Ann. Henri Poincaré 162005 (2015)

[16] R. L. Jack, P. Sollich, Eur. Phys. J. Special Topics 224 $2351(2015)$

[17] R. L. Jack, P. Sollich, J. Phys. A 47015003 (2014)

[18] V. Popkov, G. M. Schutz, J. Stat. Phys. 142627 (2011)

[19] K. Krebs, F. H. Jafarpour, G. M. Schütz, New J. Phys. 5145.1 (2003)

[20] A. C. Barato, R. Chetrite, H. Hinrichsen, D. Mukamel, J. Stat. Phys. 146294 (2012); A. C. Barato, R. Chetrite, J. Phys. A: Math. Theor 45485002 (2012)

[21] G. M. Schütz, Phase transitions and critical phenomena vol. 19 3, London: Academic (2001)

[22] H. Touchette, Phys. Rep. 4781 (2009)

[23] P. Torkamana and F. H. Jafarpour, Phys. Rev. E 87 012138 (2013)

[24] J. L. Lebowitz, H. Spohn, J. Stat. Phys. 95333 (1999); R. J. Harris, G. M. Schütz, J. Stat. Mech. P07020 (2007) 